\title{
Genetic Variations in XRCC4 (rs1805377) and ATF6 (rs2070150) are not Associated with Hepatocellular Carcinoma in Thai Patients with Hepatitis B Virus Infection
}

\author{
Jarika Makkoch ${ }^{1 \&}$, Kesmanee Praianantathavorn ${ }^{1 \&}$, Watanyoo Sopipong ${ }^{2}$, \\ Natthaya Chuaypen ${ }^{1}$, Pisit Tangkijvanich ${ }^{1,3}$, Sunchai Payungporn ${ }^{1,3 *}$
}

\begin{abstract}
The liver is one of the most common sites of cancer in the world, hepatocellular carcinoma (HCC) predominating. Chronic hepatitis B virus infection $(\mathrm{CHB})$ is considered as an important potential risk factors for HCC. Different people have diverse responses to $\mathrm{HBV}$ infection regarding the likelihood of HCC development, and host factors such as single nucleotide polymorphisms (SNPs) might account for this. The present study was conducted to evaluate any association between SNP frequencies in two genes, XRCC4 (rs1805377) and ATF6 (rs2070150), and the risk of CHB and HCC development in Thai patients. The study covered 369 subjects including 121 HCC patients, 141 with chronic hepatitis B virus infection (CHB) and 107 healthy controls. With TaqMan real-time PCR, the results showed that no significant association between XRCC4 (rs1805377) and ATF6 (rs2070150) and risk of HCC in the Thai population. From this first study of the 2 polymorphisms and HCC in Thailand it can concluded that rs1805377 and rs2070150 polymorphisms may not be applicable as genetic markers in the Thai population for HCC assessment.
\end{abstract}

Keywords: XRCC4 - rs1805377 - ATF6 - rs2070150 - hepatocellular carcinoma - SNPs - Thailand

Asian Pac J Cancer Prev, 17 (2), 591-595

\section{Introduction}

Liver cancer, ranked sixth of most common sites of cancer in world population, is originated in sites of the liver. In 2012, approximately 782,000 newly diagnosed cases occurred worldwide. Besides, about $83 \%$ of liver cancer has been arisen in under developed and developing country, which results in devastation in hospitalization and economic. Previous study showed that the highest incidence of liver cancer was in Africa and Asia, the lower incidence was presented in Europe, Latin America and Carribbean (Ferlay, 2014). One of the most frequent of liver cancer is called Hepatocellular Carcinoma (HCC), a primary liver cancer which is defined by a cancer formed within hepatocytes and further become malignant. Annually, it appears that HCC is the cause of death in 500,000-600,000 liver cancer patients (Arzumanyan et al., 2013). Previous studies showed that many risk factors (both of viral and host factors) can be responsible for HCC, such as cirrhosis, aflatoxin exposure, smoking, alcohol addiction and virus infection (Nakamoto et al., 2003; Blum, 2005; Herzer et al., 2007), especially chronic hepatitis $\mathrm{B}$ virus infection ( $\mathrm{CHB})$, which has been focused on its mechanism relevant to development of liver cancer.

Hepatitis B virus (HBV) genome consists of partially double strand DNA. The HBV virion appears in spherical shape with 40-42 $\mathrm{nm}$ in diameter. Its envelope can parcel the nucleocapsid which corporate the HBV DNA and core protein (Ganem et al., 2004; Liang, 2009). Although previous study showed that HBV infection is one of the most important factor relevant to Hepatocellular Carcinoma by incorporate its DNA to host genome, which resulted in the distortion of protein structure and activation of the hepatocyte to become malignant easier (Chemin et al., 2009), different patients have diverse capabilities to cope with HBV infection and HCC progression. Many factors can be responsible for this circumstance therefore; (1) virus factors, HBV mutation and X-protein and, (2) host factors such as sex, age and Single Nucleotide Polymorphisms (SNPs) (Chen et al., 2002; Yuen et al., 2009; Jiang et al., 2013; Subramaniam et al., 2013; Xie et al., 2013).

Single nucleotide polymorphisms (SNPs), defined by a substitution of single nucleotide base into any regions of DNA sequences which often responsible for genetic variation in human. The abnormal forms 
of SNPs on coding regions of genome can affect the aberration of protein structures and resulted in protein dysfunctions. In addition, SNPs on transcription factor sites, such as operator, promotor and enhancer can affect gene expression mechanisms while SNPs on nonprotein-coding region, encoded for RNAs that modulate the translation process, such as microRNA (miRNA) mechanism (Ramirez-Bello et al., 2013), can generate the substitute polyadenylation signals that lead to loss of microRNA regulation or generation of unspecific miRNAs (Thomas et al., 2012). Many studies showed various SNPs on human host genome that associated HCC and progression of liver cancer, such as SNPs on STAT3 gene (rs1053304) (Xie et al., 2013), STAT4 gene (re757865) (Jiang et al., 2013), SNPs rs2057482 on HIF1A gene (Guo et al., 2015) and. SNPs rs3816747 on DLC1 gene (Xie et al., 2015). Furthermore, there were 2 genes which should be considered to be candidates of SNPs associated which HCC; (1) SNPs No. rs1805377 on XRCC4, working on DNA damage and repair mechanism and; (2) SNPs No.rs2070150 on Activating Transcription factor gene called ATF6. This study aimed to evaluate the association between SNPs frequencies circumstance, which represent DNA repairing gene and activating transcription factor gene and the risk of CHB and HCC development, indicating its clinical outcomes in patients in Thailand, which can represent population in Southeast Asian country.

\section{Materials and Methods}

\section{Study population}

Three hundred and sixty-nine Thai individuals involved in this study, including 121 Hepatocellular carcinoma (HCC) patients, 141 individuals with chronic hepatitis B virus (CHB) infection and 107 healthy control subjects. Both HCC and CHB subjects were retrieved from Chulalongkorn hospital, Bangkok, Thailand whereas healthy donors from National Blood Centre Thai Red Cross Society, Bangkok, were used as healthy control subjects in this study. Subjects were classified as HCC patients by $\mathrm{CHB}$ positive, serology test and diagnostic ultrasound or/and biopsy. CHB patients had to be positive for HBsAg and anti-HBc test whereas the healthy control subjects had no record for any liver diseases and cancers including that they had to be negative against HBV and $\mathrm{HCV}$ infection. All records of individual involved in this study were retrieved from case reports including sex, age, anti-HBc, HBsAg, alanine aminotransferase (ALT) and aspartate aminotransferase (AST) Level. All subjects have been provided their information sheet and signed a consent form to commit this study. The ethical consideration and standard protocol were approved by ethical committee at faculty of Medicine, Chulalongkorn University (IRB No. 361/56)

\section{Detection of SNPS on XRCC4 (rs1805377) and ATF6 (rs2070150)}

Peripheral blood mononuclear cell (100 $\mu 1$ in volume) were obtained from each subjects for DNA extraction by phenol-chloroform: isoamyl alcohol as formerly described protocol (Sopipong et al., 2013). The NanoDrop 2000c spectrophotometry (Thermo scientific, USA) was used to measure the extracted DNA concentration and purity. SNPs on XRCC4 (rs1805377) and ATF6 (rs2070150) were determined by using a real-time qPCR. The TaqMan probe was used following the protocol for allelic discrimination assay which operated on a DreamTaq Real-Time PCR System (Thermo Scientific). PCR reaction mixture included $1 \mu 1$ of $10 \mathrm{X}$ DreamTaq Buffer (Thermo Scientific), $0.25 \mu 1$ of $40 \mathrm{X}$ primers and probe system (Thermo Scientific), $1 \mu$ of genomic DNA as template $(50-500 \mathrm{ng} / \mu \mathrm{l})$ and $7.5 \mu \mathrm{l}$ of nuclease-free water were mixed together to the final volume of $10 \mu \mathrm{l}$. The thermocycle of PCR reaction was set as follows, initial denaturation at $95^{\circ} \mathrm{C}$ for $10 \mathrm{~min}$, followed by 40 cycles of amplification including denaturation at $92^{\circ} \mathrm{C}$ for $15 \mathrm{sec}$, and the reaction was hold at $60^{\circ} \mathrm{C}$ for $1 \mathrm{~min}$ for annealing/extension. Fluorescent signals (FAM and VIC) were collected at the end of each cycle. Each experiment was conducted with positive and negative control for confirmation of interpretation. The StepOneTM software (version 2.2, Applied Biosystems) were used for allelic discrimination analysis.

\section{Statistical analysis}

The sample size in this study should be at least 53 samples for each group when the equation from previous study (Kadam et al., 2010) was used for sample size calculation. MedCalc statistical software was used to calculate the association of SNPs on XRCC4 (rs1805377) and ATF6 (rs2070150) and the HCC development using the odd ratio (OR) with $95 \%$ confidence intervals (CIs) (http://www.medcalc.org/calc/odds_ratio.php). P value < 0.05 was accepted as statistical significance.

\section{Results}

\section{Characteristics of subjects}

The characteristics of subject data in this study were found to be comparable (Table 1). The mean age of participants were 52.26, 51.75 and 51.47 years in HCC, CHB patients and healthy people respectively. The male: female ratios were presented in 8.3:1, 2.13:1 and 1.61:1 consequently. The aminotransferase (ALT) levels in HCC and CHB patients were 64.71 and $37.00 \mathrm{IU} / \mathrm{L}$ while the aspartate aminotransferase (AST) levels in those group of study were 92.34 and 32 IU/L respectively (Table 1). In addition, the total bilirubin (TB) and Albumin levels in $\mathrm{HCC}$ patients were presented at 1.16 and $0.62 \mathrm{mg} \%$ (data not shown).

\section{Genotype distributions and allele frequencies of rs1805377 on XRCC4 gene}

The genotypic distributions of rs1805377 among groups were indifferent (Table 2). HCC group ( $\mathrm{N}=121)$ contained $49.59 \%(\mathrm{~N}=60)$ of homozygous major genotype (AA), $8.26 \%(\mathrm{~N}=10)$ of homozygous minor genotype (GG) and $42.15 \%(\mathrm{~N}=51)$ of heterozygous genotype (AG). Compatibly, 44.68\% (N=63), 8.25\% $(\mathrm{N}=10)$ and $42.15 \%(\mathrm{~N}=51)$ for homozygous major genotype (AA), homozygous minor genotype (GG) and 
Lack of Association of XRCC4 (rs1805377) and ATF6 (rs2070150) SNPs with HCC in Thai Patients with HBV Infection

heterozygous genotype (AG) were reported respectively in the CHB group $(\mathrm{N}=141)$ while those in healthy group contained $51.40 \%(\mathrm{~N}=55), 9.35 \%(\mathrm{~N}=10)$ and $39.25 \%$ $(\mathrm{N}=42)$ respectively. The frequencies of the major $(\mathrm{A})$ and minor $(\mathrm{G})$ alleles were comparable among each group. Therefore; in the HCC group, major (A) and minor (G) alleles presented at $70.66 \%$ and $29.34 \%$, respectively. In the CHB group, $68.09 \%$ and $31.91 \%$ contained major (A) and minor (G) alleles, respectively while these two alleles in healthy group showed in $71.03 \%$ and $28.97 \%$ consequently (Table 2).

Genotype distributions and allele frequencies of rs2070150 on ATF6 gene

The genotypic distributions of rs2070150 on ATF6 gene among groups were found to be also indifferent (Table 3). Homozygous major genotype (CC) was presented at $33.88 \%, 43.97 \%$ and $42.06 \%$ in $\mathrm{HCC}, \mathrm{CHB}$ and healthy control respectively. Moreover, the (GG) homozygous minor genotype was detected in HCC (14.88\%), CHB (9.93\%) and healthy control (9.35\%) while the heterozygous genotype (CG) was detected in percentage of 51.24, 46.1 and 48.6 in HCC patients, CHB patients and healthy control subjects respectively. The allele frequencies were found not to show statistical difference among groups of study. The major (C) alleles presented in HCC (59.5\%), CHB (67.02\%) and healthy control $(66.36 \%)$ while the minor $(\mathrm{G})$ allele was detected in $\mathrm{HCC}, \mathrm{CHB}$ and healthy control as $40.5 \%, 32.98 \%$ and $33.64 \%$, respectively (Table 3 ).

Analysis of SNPs (rs1805377) on XRCC4 gene, (rs2070150) ATF6 gene and their association with HBVrelated $\mathrm{HCC}$

Compare to $\mathrm{CHB}+$ healthy group and each of $\mathrm{CHB}$ group and healthy group, the genotypes of XRCC4 gene (rs1805377) and ATF6 gene (rs2070150) were not considerably associated ( $\mathrm{P}$ value $>0.05$ ) with the progression of HCC among Thai population. After adjusting for variables such as age and sex in the logistic regression analysis, minor allele of rs1805377 and rs2070150 were not significantly correlated ( $\mathrm{P}$ value $>$ $0.05)$ with the development of HCC in Thai population. The overall odd ratio $(95 \% \mathrm{CI})$ and $\mathrm{P}$ value were showed in table 2 and 3.

\section{Discussion}

This study aimed to evaluate the association between single nucleotide polymorphisms (SNPs) on XRCC4 gene (rs 1805377) and ATF6 gene (rs2070150) and the incidence of primary liver cancer type HCC (hepatocellular carcinoma) and its progression from CHB (Chronic Hepatitis B) to become HCC in Thai Population. The XRCC4, mapped on chromosome 5 in position $5 q 13-14$

Table 1. Characteristics of the Subjects in the Study

\begin{tabular}{lccccc}
\hline Characteristics & $\begin{array}{c}\text { HCC cases } \\
\text { N=121 }(\%)\end{array}$ & $\begin{array}{c}\text { CHB subjects } \\
\text { N=141 }(\%)\end{array}$ & $\begin{array}{c}\text { P value } \\
(\text { CHB vs HCC) }\end{array}$ & $\begin{array}{c}\text { Healthy subjects } \\
\text { N=107 }(\%)\end{array}$ & $\begin{array}{c}\text { P value } \\
\text { (HCC vs Healthy) }\end{array}$ \\
\hline Mean Age (years) & $52.26 \pm 7.57$ & $51.75 \pm 9.09$ & 0.6255 & $51.47 \pm 3.77$ & 0.3294 \\
Male (\%) & $108(89.26 \%)$ & $96(68.09 \%)$ & & $66(61.68 \%)$ & \\
AST (IU/L) & $92.34 \pm 80.45$ & $32 \pm 23.2$ & $<0.0001$ & $(\mathrm{NA})$ & (NA) \\
ALT (IU/L) & $64.71 \pm 62.23$ & $37 \pm 41.23$ & $<0.0001$ & (NA) & (NA) \\
\hline
\end{tabular}

Table 2. Genotype Distributions and Allele Frequencies of rs1805377 on XRCC4 Gene

\begin{tabular}{|c|c|c|c|c|c|c|c|}
\hline \multirow{2}{*}{$\begin{array}{l}\text { SNP } \\
\text { rs1805377 }\end{array}$} & \multirow{2}{*}{$\begin{array}{c}\text { HCC } \\
\mathrm{N}=121\end{array}$} & \multirow{2}{*}{$\begin{array}{c}\text { CHB } \\
N=141\end{array}$} & \multirow{2}{*}{$\begin{array}{l}\text { Healthy } \\
N=107\end{array}$} & \multicolumn{2}{|c|}{$\mathrm{HCC}$ vs $\mathrm{CHB}$} & \multicolumn{2}{|c|}{ HCC vs Healthy } \\
\hline & & & & OR $(95 \% \mathrm{CI})$ & $P$ values & OR $(95 \% \mathrm{CI})$ & Pvalues \\
\hline \multicolumn{8}{|l|}{ Genotype } \\
\hline AA & $60(49.59 \%)$ & $63(44.68 \%)$ & $55(51.40 \%)$ & 1 & - & 1 & - \\
\hline $\mathrm{AG}$ & $66(46.81 \%)$ & $51(42.15 \%)$ & $42(39.25 \%)$ & $0.8114(0.4880-1.3491)$ & 0.4204 & $1.1131(0.6433-1.9261)$ & 0.7017 \\
\hline GG & $12(8.51 \%)$ & $10(8.26 \%)$ & $10(9.35 \%)$ & $0.875(0.3520-2.1752)$ & 0.7738 & $0.9167(0.3546-2.3699)$ & 0.8575 \\
\hline $\mathrm{AG}+\mathrm{GG}$ & - & - & - & $0.8212(0.5045-1.3365)$ & 0.4278 & $1.0753(0.6391-1.8093)$ & 0.7844 \\
\hline \multicolumn{8}{|l|}{ Allele } \\
\hline Major A & $171(70.66 \%)$ & $192(68.09 \%)$ & $152(71.03 \%)$ & 1 & - & 1 & - \\
\hline Minor G & $71(29.34 \%)$ & $90(31.91 \%)$ & $62(28.97 \%)$ & $0.8858(0.6099-1.2864)$ & 0.5241 & $1.0179(0.6791-1.5258)$ & 0.9315 \\
\hline
\end{tabular}

Table 3. Genotype Distributions and Allele Frequencies of rs2070150 on ATF6 Gene

\begin{tabular}{|c|c|c|c|c|c|c|c|}
\hline \multirow{2}{*}{$\begin{array}{l}\text { SNP } \\
\text { rs2070150 }\end{array}$} & \multirow{2}{*}{$\begin{array}{c}\mathrm{HCC} \\
\mathrm{N}=121\end{array}$} & \multirow{2}{*}{$\begin{array}{c}\mathrm{CHB} \\
\mathrm{N}=141\end{array}$} & \multirow{2}{*}{$\begin{array}{l}\text { Healthy } \\
\mathrm{N}=107\end{array}$} & \multicolumn{2}{|c|}{$\mathrm{HCC}$ vs $\mathrm{CHB}$} & \multicolumn{2}{|c|}{ HCC vs Healthy } \\
\hline & & & & OR $(95 \% \mathrm{CI})$ & $\overline{\mathrm{P} \text { values }}$ & OR (95\% CI) & Pvalues \\
\hline \multicolumn{8}{|l|}{ Genotype } \\
\hline $\mathrm{CC}$ & $41(33.88 \%)$ & $62(43.97 \%)$ & $45(42.06 \%)$ & 1 & - & 1 & - \\
\hline $\mathrm{CG}$ & $62(51.24 \%)$ & $65(46.1 \%)$ & $52(48.6 \%)$ & $1.4424(0.8524-2.4409)$ & 0.1723 & $1.3086(0.7466-2.2937)$ & 0.3475 \\
\hline GG & $18(14.88 \%)$ & $14(9.93 \%)$ & $10(9.35 \%)$ & $1.9443(0.8717-4.3364)$ & 0.1043 & $0.9167(0.3546-2.3699)$ & 0.1299 \\
\hline $\mathrm{CG}+\mathrm{GG}$ & - & - & - & $1.5313(0.9267-2.5306)$ & 0.0963 & $1.0753(0.6391-1.8093)$ & 0.2046 \\
\hline \multicolumn{8}{|l|}{ Allele } \\
\hline Major C & $144(59.5 \%)$ & $189(67.02 \%)$ & $142(66.36 \%)$ & 1 & - & 1 & - \\
\hline Minor G & $98(40.5 \%)$ & $93(32.98 \%)$ & $72(33.64 \%)$ & $1.3831(0.9677-1.9766)$ & 0.0751 & $1.3422(0.9156-1.9676)$ & 0.1315 \\
\hline
\end{tabular}


(Jung et al.,2012), was found to encode the protein crucial for DNA repair mechanisms by functioning together with DNA ligase IV and the DNA-dependent protein kinase proteins whose mechanism is normally required for normal development process and tumor suppression mechanisms. From this characteristic, the gene XRCC4 was considered to be one of candidates SNPs to control the HCC due to the suggestion from previous study that the Hepatitis virus can cause DNA damage in host genome, leading to the major factor to develop the progression of CHB to become HCC (Jung et al., 2012). In Korea, previous study suggested that the SNPs on XRCC4 can be related to the significantly increased overall survival in HCC patient population with CHB and affected the clinical outcomes (Jung et al., 2012). Furthermore, XRCC4 (rs1805377) has been reported to has significant association with risk of non-Hodgkin lymphoma (OR (95\% CI) 2.07(1.10-3.90)) (Guo et al., 2014), glioma (OR (95\% CI) 1.77(1.12-2.80)) (Zhao et al., 2013), lung cancer $(\mathrm{p}=0.015, \mathrm{OR}=2.67)$ (Tseng et al., 2009), renal cell carcinoma (OR (95\% CI) 1.56 (1.082.26) (Margulis et al., 2008), bladder cancer (OR (95\% CI) 1.33 (1.08-1.64)) (Figueroa et al., 2007) and breast cancer (Fu et al., 2003). Even if rs1805377 does not influence susceptibility to prostate cancer, it still has significant risk with high Gleason grade ( $\mathrm{p}=0.028, \mathrm{OR}=2.87$ ) (Mandal, 2011). On the other hand, some other studies indicated in contrast, SNP rs 1805377 does not associated. There was no difference in distribution of rs1805377 genotypes among esophageal squamous cell carcinoma patients and controls (Yang et al., 2015). No association was found between variants of XRCC4 rs1805377 and development of glioma in Chinese population (Youle et al., 2015). In Mittal RD study, rs 1805377 does not have significant different distributions in the frequency between urothelial bladder cancer patients and controls (Mittal et al., 2012). In Taiwan, neither Tesng HC's study, nor Chiu CF's have found statistical significant association between this SNP and oral cancer risk (Chiu et al., 2008; Tseng et al., 2008).

Activating transcription factor 6 (ATF6) gene, located on chromosome 1 at q22 position and organized into 16 exons (So et al., 2009), involves in many cellular mechanisms, such as cell differentiation, inflammation and apoptosis. In addition, ATF6 usually acts as a motivator to unfold many proteins in endoplasmic reticulum to reduce cellular stress caused by unfolded protein response (UPR). The upregulation control of genes generating the UPR can provide the advantage to the tumor cells growth and important to cancer development (Fels et al., 2006; Suh et al., 2012). Previous study found that the HCC high frequencies of protective allele C of rs2070150 on ATF6 gene was associated with higher ATF6 expression and can function of stimulating downstream genes to prevent liver cancer (Wu et al., 2014). However, some study reveal the contrast of their finding, when the eminence of ATF6 mRNAs was found in hepatocarcinogenesis (Shuda et al., 2003).

Results showed that there were no significant associations between rs 1805377 and rs2070150 polymorphisms with the risk of HCC in Thai population, which can be represented the genetic distribution in Southeast Asia region. Therefore, it can concluded from this study that the SNPs rs1805377 and rs2070150 polymorphisms may not be able to use as a genetic marker in Thai population in HCC assessment. This study had the limitation in unmatched gender among each group, however, the incident of HCC showed a lot more frequent in male rather than female gender. Therefore, further case-control study with gender matched among each group is required in order to control confounding factors and confirm the association of rs1805377 and rs2070150 polymorphisms with the susceptibility HCC in Thai population.

\section{Acknowledgements}

This research has been supported by the Ratchadaphiseksomphot Endowment Fund of Chulalongkorn University (CU-57-001-HR) and the Postdoctoral Fellowship from Chulalongkorn University.

\section{References}

Arzumanyan A, Reis HM, Feitelson MA (2013). Pathogenic mechanisms in HBV- and HCV associated hepatocellular carcinoma. Nat Rev Cancer, 13, 123-35.

Blum HE (2005). Hepatocellular carcinoma: therapy and prevention. World J Gastroenterol, 11, 7391-400.

Chemin I, Zoulim F (2009). Hepatitis B virus induced hepatocellular carcinoma. Cancer Lett, 286, 52-9.

Chen CJ, Chen DS (2012). Interaction of hepatitis B virus, chemical carcinogen, and genetic susceptibility: multistage hepatocarcinogenesis with multifactorial etiology. Hepatology, 36, 1046-9.

Chiu CF, Tsai MH, Tseng HC, et al (2008). A novel single nucleotide polymorphism in XRCC4 gene is associated with oral cancer susceptibility in Taiwanese patients. Oral Oncol, 44, 898-902.

Fels DR, Koumenis C (2006). The PERK/eIF2alpha/ATF4 module of the UPR in hypoxia resistance and tumor growth. Cancer Biol Ther, 5, 723-8.

Ferlay J, Soerjomataram I, Ervik M, et al (2014). Cancer Incidence and Mortality Worldwide. In 'Globocan': International Agency for Research on Cancer, Lyon, France 2012 v1.1.

Figueroa JD, Malats N, Rothman N, et al (2007). Evaluation of genetic variation in the double-strand break repair pathway and bladder cancer risk. Carcinogenesis, 28, 1788-93.

Fu YP, Yu JC, Cheng TC, et al (2003). Breast cancer risk associated with genotypic polymorphism of the nonhomologous end-joining genes: a multigenic study on cancer susceptibility. Cancer Res, 63, 2440-6.

Ganem D, Prince AM (2004). Hepatitis B virus infection-natural history and clinical consequences. $N$ Engl J Med, 350, 1118-29.

Guo H, Bassig BA, Lan Q, et al (2014). Polymorphisms in DNA repair genes, hair dye use, and the risk of non-Hodgkin lymphoma. Cancer Causes Control, 25, 1261-70.

Guo X, Li D, Chen Y, et al (2015). SNP rs2057482 in HIF1A gene predicts clinical outcome of aggressive hepatocellular carcinoma patients after surgery. Sci Rep, 5, 11846.

Herzer K, Sprinzl MF, Galle PR (2007). Hepatitis viruses: live and let die. Liver Int, 27, 293-301.

Jiang DK, Sun J, Cao G, et al (2013). Genetic variants in STAT4 and HLA-DQ genes confer risk of hepatitis B virus-related hepatocellular carcinoma. Nat Genet, 45, 72-5.

Jung SW, Park NH, Shin JW, et al (2012). Polymorphisms 
Lack of Association of XRCC4 (rs1805377) and ATF6 (rs2070150) SNPs with HCC in Thai Patients with HBV Infection

of DNA repair genes in Korean hepatocellular carcinoma patients with chronic hepatitis B: possible implications on survival. J Hepatol, 57, 621-7.

Liang TJ (2009). Hepatitis B: the virus and disease. Hepatology, 49, 13-21.

Mandal RK, Singh V, Kapoor R, et al (2011). Do polymorphisms in XRCC4 influence prostate cancer susceptibility in North Indian population? Biomarkers, 16, 236-42.

Margulis V, Lin J, Yang H, et al (2008). Genetic susceptibility to renal cell carcinoma: the role of DNA double-strand break repair pathway. Cancer Epidemiol Biomarkers Prev, 17, 2366-73.

Nakamoto Y, Kaneko S (2003). Mechanisms of viral hepatitis induced liver injury. Curr Mol Med, 3, 537-44.

Shuda M, Kondoh N, Imazeki N, et al (2003). Activation of the ATF6, XBP1 and grp78 genes in human hepatocellular carcinoma: a possible involvement of the ER stress pathway in hepatocarcinogenesis. J Hepatol, 38, 605-14.

So AY, de la Fuente E, Walter P, et al (2009). The unfolded protein response during prostate cancer development. Cancer Metastasis Rev, 28, 219-23.

Sopipong W, Tangkijvanich P, Payungporn S, et al (2013). The KIF1B (rs17401966) single nucleotide polymorphism is not associated with the development of HBV-related hepatocellular carcinoma in Thai patients. Asian Pacific $J$ Cancer Prev, 14, 2865-9.

Subramaniam A, Shanmugam MK, Perumal E, et al (2013). Potential role of signal transducer and activator of transcription (STAT) 3 signaling pathway in inflammation, survival, proliferation and invasion of hepatocellular carcinoma. Biochim Biophys Acta, 1835, 46-60.

Suh DH, Kim MK, Kim HS, et al (2012). Unfolded protein response to autophagy as a promising druggable target for anticancer therapy. Ann N Y Acad Sci, 1271, 20-32.

Thomas LF, Sætrom P (2012). Single nucleotide polymorphisms can create alternative polyadenylation signals and affect gene expression through loss of MicroRNA-Regulation. PLoS Comput Biol, 8, 1002621.

Tseng HC, Tsai MH, Chiu CF, et al (2008). Association of XRCC4 codon 247 polymorphism with oral cancer susceptibility in Taiwan. Anticancer Res, 28, 1687-91.

Tseng RC, Hsieh FJ, Shih CM, et al (2009). Lung cancer susceptibility and prognosis associated with polymorphisms in the nonhomologous end-joining pathway genes: a multiple genotype-phenotype study. Cancer, 115, 2939-48.

Wu X, Xin Z, Zhang W, et al (2014). A missense polymorphism in ATF6 gene is associated with susceptibility to hepatocellular carcinoma probably by altering ATF6 level. Int J Cancer, 135, 61-8.

Xie CR, Sun HG, Sun Y, et al (2015). Significance of genetic variants in DLC1 and their association with hepatocellular carcinoma. Mol Med Rep, 12, 4203-9.

Xie J, Zhang Y, Zhang Q, et al (2013). Interaction of signal transducer and activator of transcription 3 polymorphisms with hepatitis B virus mutations in hepatocellular carcinoma. Hepatology, 57, 2369-77.

Yang HL, Qiao DD, Li K, et al (2015). Association of genetic polymorphisms in PRKDC and XRCC4 with risk of ESCC in a high-incidence region of North China. Tumori. (Epub ahead of print)

Youle Su, Songtao Qi, Changwu Dou, et al (2015). Association of LIG4 and XRCC4 gene polymorphisms with the risk of human glioma in a Chinese population. Int J Clin Exp Pathol, 8, 2057-62.

Yuen MF, Hou JL, Chutaputti A (2009). Hepatocellular carcinoma in the Asia pacific region. J Gastroenterol Hepatol, 24, 346-53.
Zhao P, Zou P, Zhao L, et al (2013). Genetic polymorphisms of DNA double-strand break repair pathway genes and glioma susceptibility. BMC Cancer, 13, 234. 